\title{
Inhaled NO accelerates restoration of liver function in adults following orthotopic liver transplantation
}

\author{
John D. Lang Jr., ${ }^{1}$ Xinjun Teng,, ${ }^{2}$ Phillip Chumley, ${ }^{3}$ Jack H. Crawford, ${ }^{2,3}$ T. Scott Isbell, ${ }^{2}$ \\ Balu K. Chacko, ${ }^{2}$ Yuliang Liu, ${ }^{1}$ Nirag Jhala, ${ }^{2}$ D. Ralph Crowe, ${ }^{2}$ Alvin B. Smith, ${ }^{3}$ Richard C. Cross, ${ }^{3}$ \\ Luc Frenette, ${ }^{3}$ Eric E. Kelley, ${ }^{4}$ Diana W. Wilhite, ${ }^{3}$ Cheryl R. Hall, ${ }^{3}$ Grier P. Page, ${ }^{5}$ Michael B. Fallon, ${ }^{6}$ \\ J. Steven Bynon, ${ }^{7}$ Devin E. Eckhoff, ${ }^{7}$ and Rakesh P. Patel ${ }^{2,8}$

\begin{abstract}
${ }^{1}$ Department of Anesthesiology, University of Washington School of Medicine, Seattle, Washington, USA. '2Department of Pathology and ${ }^{3}$ Department of Anesthesiology, University of Alabama at Birmingham, Birmingham, Alabama, USA. ${ }^{4}$ Department of Anesthesiology, University of Pittsburgh School of Medicine, Pittsburgh, Pennsylvania, USA. ${ }^{5}$ Department of Biostatistics, ${ }^{6}$ Department of Medicine, ${ }^{7}$ Department of Surgery, and ${ }^{8}$ Center for Free Radical Biology, University of Alabama at Birmingham, Birmingham, Alabama, USA.
\end{abstract}

\begin{abstract}
Ischemia/reperfusion (IR) injury in transplanted livers contributes to organ dysfunction and failure and is characterized in part by loss of NO bioavailability. Inhalation of NO is nontoxic and at high concentrations $(80 \mathrm{ppm})$ inhibits IR injury in extrapulmonary tissues. In this prospective, blinded, placebo-controlled study, we evaluated the hypothesis that administration of inhaled NO (iNO; $80 \mathrm{ppm}$ ) to patients undergoing orthotopic liver transplantation inhibits hepatic IR injury, resulting in improved liver function. Patients were randomized to receive either placebo or iNO ( $n=10$ per group) during the operative period only. When results were adjusted for cold ischemia time and sex, iNO significantly decreased hospital length of stay, and evaluation of serum transaminases (alanine transaminase, aspartate aminotransferase) and coagulation times (prothrombin time, partial thromboplastin time) indicated that iNO improved the rate at which liver function was restored after transplantation. iNO did not significantly affect changes in inflammatory markers in liver tissue 1 hour after reperfusion but significantly lowered hepatocyte apoptosis. Evaluation of circulating NO metabolites indicated that the most likely candidate transducer of extrapulmonary effects of iNO was nitrite. In summary, this study supports the clinical use of iNO as an extrapulmonary therapeutic to improve organ function following transplantation.
\end{abstract}

\section{Introduction}

Liver ischemia with consequent reperfusion results in a multitude of cellular, humoral, and biochemical events leading to hepatocellular injury and dysfunction $(1,2)$. Hepatic ischemia/reperfusion (IR) injury is a significant complication in liver transplantation that can predispose patients to a profound reperfusion syndrome, resulting in primary graft nonfunction and initial poor function of the graft. In addition, increased susceptibility of marginal livers to IR injury limits the number available for transplantation $(3,4)$. Pharmacological approaches to curtailing the perturbations of liver IR during allograft transplantation have generally been unsuccessful due in large part to the complex mechanisms involved (5). Experimental studies of hepatic IR injury indicate roles for infiltrating polymorphonuclear cells (PMNs) and $\mathrm{T}$ cells, activation of Kupffer cells and endothelial cells, and formation of ROS/reactive nitrogen species (ROS/RNS) $(1,2,6)$. This complexity arises in part from the involvement of different mediators and cell types at temporally distinct stages of the injury response and the nature of the experimental model studied (species, sex, etc.).

Nonstandard abbreviations used: ALT, alanine transaminase; AST, aspartate aminotransferase; BD1, blood draw $1 ; \mathrm{HbNO}$, nitrosylhemoglobin; iNO, inhaled NO; IR, ischemia/reperfusion; MPO, myeloperoxidase; NOx, NO derivative(s); PMN, polymorphonuclear cell; PT, prothrombin time; PTT, partial thromboplastin time; RNS, reactive nitrogen species; $\mathrm{SNO}, \mathrm{S}$-nitrosothiols; $\mathrm{XNO}, \mathrm{C}$ - or $\mathrm{N}$-nitrosamines; $\mathrm{XOR}$, xanthine oxidoreductase.

Conflict of interest: The authors have declared that no conflict of interest exists. Citation for this article: J. Clin. Invest. 117:2583-2591 (2007). doi:10.1172/JCI31892.
Irrespective of the precise mechanisms involved, increased inflammation and cytotoxicity are key components in hepatocellular dysfunction during the pathogenesis of liver IR injury and provide targets for therapeutic interventions.

Recently, decreased hepatic production of NO from eNOS (also known as NOS3) within 1 hour of reperfusion in humans undergoing orthotopic liver transplantation was suggested to contribute to the IR-dependent injury observed (7). Moreover, studies in mice have shown that administration of NO-donors or overexpression of hepatic eNOS inhibits IR injury in the liver $(8,9)$. Thus, replenishing $\mathrm{NO}$ via therapeutic administration may serve to abrogate reperfusion injury in these vulnerable patients. NO-mediated protection in IR injury can occur via multiple mechanisms, including cytoprotection, antiinflammatory effects, modulation of mitochondrial respiration, antioxidant effects, and maintenance of vasomotor tone at the presinusoidal site within the hepatic sinusoid. In contrast, NO can also contribute to IR injury via formation of secondary RNSs including peroxynitrite (9).

Inhaled NO (iNO) has been used clinically for nearly 2 decades for the treatment of reduced oxygen tensions and reduced pulmonary artery pressures in patients suffering from inflammatory-mediated lung injury, and to assist in enhancing flow in ventricular assist devices. Unfortunately, its use in adults has met with limited success, as the clinical evidence does not support its administration as a first-line therapeutic agent for pulmonaryrelated diseases (10). Traditional thinking has been that as iNO crosses the alveolar-capillary membrane, it is rendered inactive by 


\section{Table 1}

Patient characteristics and demographics

\begin{tabular}{|c|c|c|c|}
\hline & Placebo $(n=10)$ & iNO $(n=10)$ & $P$ \\
\hline Age (yr) & $51(34-67)$ & $53(30-61)$ & 0.73 \\
\hline Sex & 5 males, 5 females & 7 males, 3 females & \\
\hline Weight (kg) & $102(60-134)$ & $85(51-140)$ & 0.25 \\
\hline Race & 8 white, 2 African American & 10 white & \\
\hline MELD & $19(6-35)$ & $18.5(13-25)$ & 0.47 \\
\hline APACHE II & $9.5(2-24)$ & $13(7-28)$ & 0.2 \\
\hline ASA classification & 4 & 4 & 1 \\
\hline Cold ischemic time (min) & $363(112-676)$ & $404(69-722)$ & 0.94 \\
\hline Total surgical time $(\mathrm{h})$ & $4(3.4-5.2)$ & $4(3.6-4.7)$ & 0.96 \\
\hline Portal vein clamp time (min) & $49(43-66)^{A}$ & $49.5(35-81)$ & 0.75 \\
\hline $\begin{array}{l}\text { Time between portal vein unclamp } \\
\text { and hepatic artery unclamp (min) }\end{array}$ & $19(0-67)$ & $26.5(2-35)$ & 0.38 \\
\hline Allograft steatosis (no.) & $\leq 5 \%$ for $10 / 10$ & $\leq 5 \%$ for $10 / 10$ & \\
\hline Preservation solution & HTK for $9 / 10$; UW for $1 / 10$ & HTK for $7 / 10$; UW for $3 / 10$ & \\
\hline Donor age (yr) & $33.5(18-60)$ & $23.5(19-53)$ & 0.37 \\
\hline Indications for transplantation (no.) & $\begin{array}{c}\text { Nonalcoholic steatohepatitis (3), } \\
\text { hepatitis C (2), Laennec cirrhosis (1), } \\
\text { autoimmune hepatitis (1), hemochromatosis (1), } \\
\alpha-1 \text { antitrypsin deficiency (1), } \\
\text { hepatocellular cancer/hemochromatosis (1) }\end{array}$ & $\begin{array}{c}\text { Nonalcoholic steatohepatitis (1), } \\
\text { hepatitis C (4), } \\
\text { hepatitis B (2), } \\
\text { cryptogenic cirrhosis (3) }\end{array}$ & \\
\hline Postoperative complications (no.) & $\begin{array}{c}\text { Retransplant (1), pneumothorax (1), } \\
\text { hydrothorax (1) }\end{array}$ & $\begin{array}{l}\text { Pneumonia (1), } \\
\text { Respiratory failure (1) }\end{array}$ & \\
\hline
\end{tabular}

APACHE, acute physiology and chronic health evaluation; ASA, American Society of Anesthesiology; HTK, histidine-tryptophan-ketoglutarate; MELD, model for end-stage liver disease; UW, University of Wisconsin. Values show median (range). $P$ values calculated from unpaired $t$ test. A $n=9$.

rapid reactions with oxy- or deoxyhemoglobin in the rbc. However, seminal studies by Kubes et al. dismissed this concept, demonstrating that iNO possesses extrapulmonary bioactivity in the mesenteric vasculature by preventing neutrophil adhesion in a feline model of IR injury (11). These concepts have been extended to show that iNO inhibits myocardial IR injury in mice (12), inhibits myocardial injury in patients undergoing cardiopulmonary bypass (13), improves forearm blood flow in healthy volunteers (14), and inhibits IR-dependent inflammatory injury in patients undergoing knee surgery (15). How iNO mediates extrapulmonary effects remains unclear, with the general hypothesis being that iNO forms a relatively stable, $\mathrm{NO}$-containing intermediate in the circulation, which then mediates systemic effects either directly or after being recycled to NO. Recent evidence in a feline model of IR suggests that the intermediate may be plasma $S$-nitrosothiols (SNO) (e.g., $S$-nitrosoalbumin) (16), whereas studies in humans and mice indicate nitrite as a possible mediator $(12,14)$. A key role for nitrite is also indicated by its direct administration protecting against hepatic and myocardial IR in murine models and the description of biological mechanisms for nitrite reduction to NO under ischemic conditions (17-20). It should be noted that other NO-containing candidates in the circulation that are relatively labile under biological conditions may also be formed upon NO inhalation (via nitrosylation or $S$-nitrosation reactions). These include include $\mathrm{SNO}$ in the rbc, ferrous nitrosylhemoglobin ( $\mathrm{HbNO}$ ), and C- or $\mathrm{N}$-nitrosamines (referred to as XNO) $(20,21)$.

In this study, we hypothesized that preemptive administration of iNO to patients undergoing orthotropic liver transplantation would decrease IR-induced injury via an antiinflammatory mechanism. This hypothesis was tested via a prospective, blinded, randomized placebo-controlled trial. We found that patients receiving
iNO had improved hepatic function after transplantation, which was associated with inhibition of hepatic cell death, with little effect on PMN accumulation. In addition, measurement of different NO derivatives suggests that the beneficial effects of iNO may occur via increasing circulating levels of nitrite.

\section{Results}

Evaluation of $i N O$ safety and efficacy. Table 1 displays the patient demographics. No differences in recipient age, clinical indication for transplantation, liver fat content, cold ischemic time for donor liver, or time of surgery between placebo and iNO groups were observed. No differences in perioperative use of immunosuppressive drugs were observed (Supplemental Table 1; supplemental material available online with this article; doi:10.1172/ JCI31892DS1). iNO was administered according to the study protocol shown in Figure 1A and described in Methods. Primary clinical concerns surrounding the use of iNO included exposure to $\mathrm{NO}_{2}$, formation of methemoglobin (metHb), and inhibition of platelet function. $\mathrm{NO}_{2}$ levels increased from $0.31 \pm 0.11$ (placebo) to $0.75 \pm 0.13 \mathrm{ppm}$ (iNO) (mean $\pm \mathrm{SEM} ; n=10$ ) as measured 1 hour after reperfusion. Figure $1 \mathrm{~B}$ shows that although metHb levels rose continuously during iNO administration, they only reached approximately $2 \%$, a nontoxic level, by the end of the protocol. Interestingly, the volume of transfused platelets required during the surgical procedure was significantly reduced, by approximately $50 \%$ in the iNO group, suggesting that the therapy had no apparent adverse effects on platelet function (Figure 1C). A similar trend towards reduced rbc transfusion in the iNO group was also observed (data not shown). Finally, no differences in cardiac or lung-related complications between groups were observed (Table 1). The efficacy of iNO administration in stimulating NO-dependent 


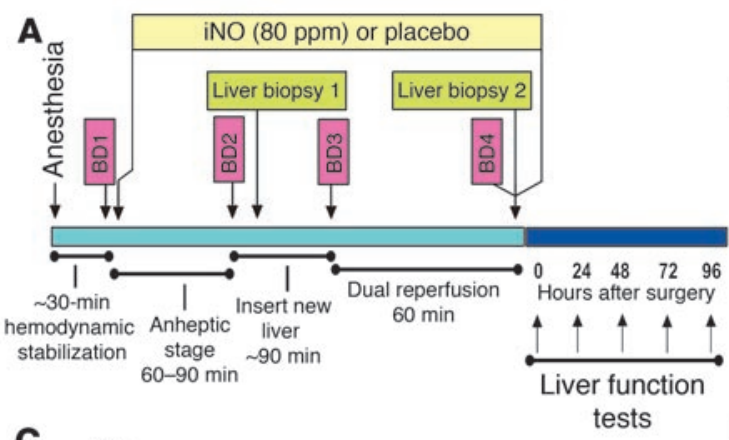

C

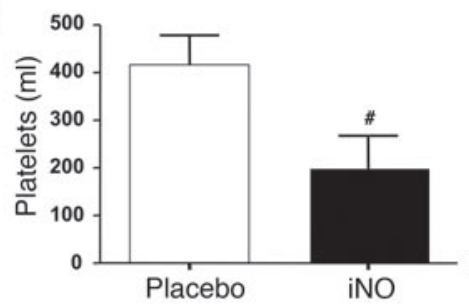

E

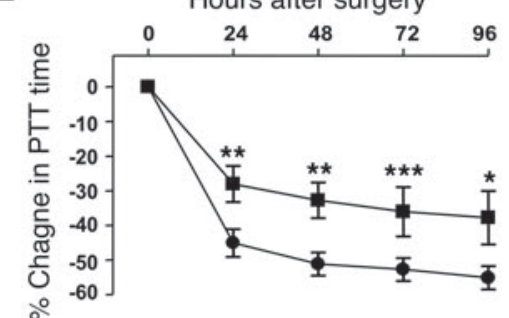

G

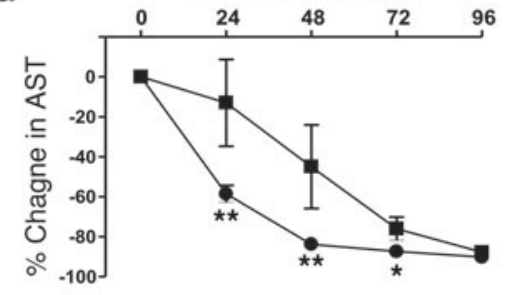

\section{B}

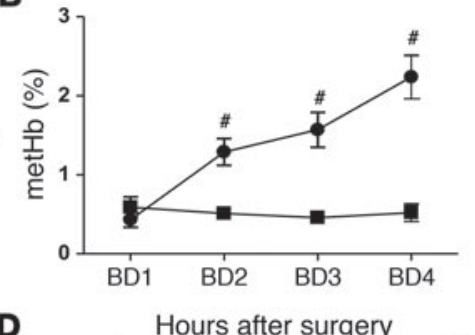

D

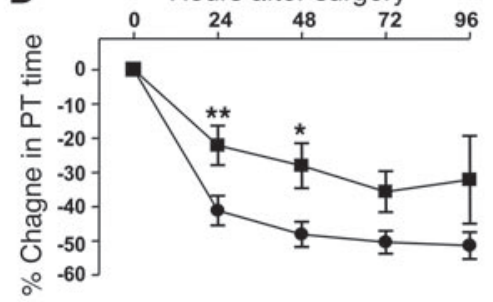

F

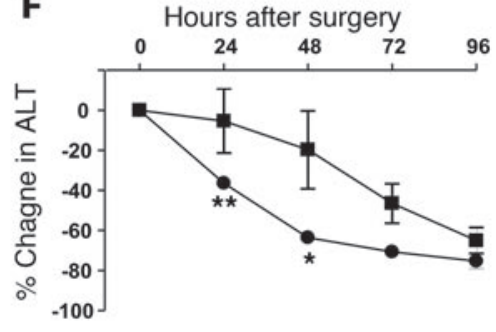

H

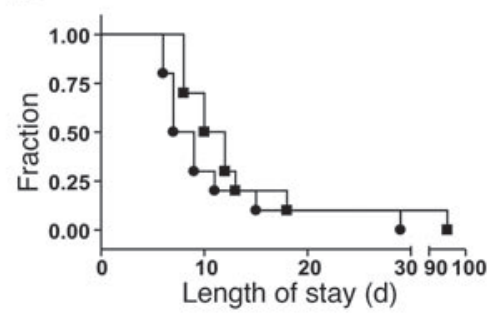

\section{Figure 1}

iNO therapy and human liver transplantation. (A) Experimental protocol for administering placebo or iNO to patients and sample (blood and liver biopsy) collection. (B) Methemoglobin (metHb) levels as a function of blood draw. ${ }^{\#} \leq 0.001$ for corresponding placebo versus iNO measurements by unpaired $t$ test. (C) Volume of platelets transfused during surgery ${ }^{\#} P \leq 0.05$. (D and E) Average percent decrease in PT and PTT after surgery. Data are normalized to coagulation times measured immediately ( $<1$ hour) after surgery and were $26.7 \pm 1.4$ seconds (placebo) and $34.4 \pm 2.5$ seconds (iNO) for PT and $54.6 \pm 7.3$ seconds (placebo) and $70.2 \pm 6.5$ seconds (iNO) for PTT. (F and G) Average percent decrease in serum ALT and AST levels after surgery. Data were normalized to ALT and AST levels measured immediately ( $<1$ hour) after surgery and were $601.8 \pm 145.4 \mathrm{U} / \mathrm{l}$ (placebo) and $689.3 \pm 149.5 \mathrm{U} / \mathrm{l}$ (iNO) for ALT and $922.1 \pm 228.7 \mathrm{U} / \mathrm{l}$ (placebo) and $940.9 \pm 211.3 \mathrm{U} / \mathrm{l}$ (iNO) for AST. For data in panels $\mathbf{D}-\mathbf{G},{ }^{*} P \leq 0.05$, ${ }^{\star \star} P \leq 0.01,{ }^{* \star \star} P \leq 0.001$ for corresponding placebo versus iNO measurements. (H) Cox analysis of patient hospital length of stay. $P=0.034$ adjusted for sex and cold ischemic time. Filled squares: placebo; filled circles: iNO. signaling was indicated by significant increases in plasma cGMP in patients receiving iNO (Supplemental Figure 1A).

iNO improves posttransplantation liver function tests and decreases patient hospital length of stay. Liver function was evaluated by measuring prothrombin time (PT) and partial thromboplastin time (PTT) (for assessment of extrinsic and intrinsic coagulation pathways, respectively) and measurement of serum alanine transaminase (ALT) and aspartate aminotransferase (AST) at 24-hour intervals after transplantation. Figure 1, D and E, shows the average percent changes in PT and PTT relative to measurements taken immediately ( $<1$ hour) after surgery. PT and PTT decreased as a function of time after surgery in both placebo and iNO patients; however, the magnitude of this change was greater in iNO patients, suggesting that iNO results in improved liver function after transplantation. This conclusion is further indicated in Figure 1, F and G, which show changes in ALT and AST, respectively. In the placebo group, ALT and AST did not change over the initial 24-hour interval, but they then gradually declined in a time-dependent manner. In the iNO group, however, ALT and AST levels decreased significantly within 24 hours, with the trend toward a greater decrease in AST and ALT in the iNO compared with the placebo group sustained until 72-96 hours. Note that absolute concentrations of ALT and AST immediately after surgery did not differ between the placebo and iNO groups (Figure 1 legend). The observed improvement in liver function tests in patients receiving iNO was associated with a significant decrease in patient hospital length of stay (i.e., time to discharge). Figure $1 \mathrm{H}$ shows results from a Cox proportional hazard analysis indicating that iNO reduced hospital length of stay by 1.24 days $(P=0.034)$ when adjusted for sex and cold ischemic time (sex $[P=0.036]$ : males 1.63 days less than females; and cold ischemic time $[P=0.022]$ : increase of $0.002 \mathrm{~d} / \mathrm{min}$ cold ischemic time). Supplemental Figure 1 plots the length of stay versus cold ischemic time and illustrates the concept that for a given cold ischemic time, patients receiving iNO have a lower length of stay. Finally, at 6 months after surgery, 2 of 10 placebo patients and 3 of 10 iNO patients had a biopsy-proven rejection episode, 1 of which was thought secondary to noncompliance with the prescribed medicine regime. Additional morbidity included sepsis $(n=1)$, organ failure $(n=2)$, and recurrent hepatitis C $(n=2)$. 
A

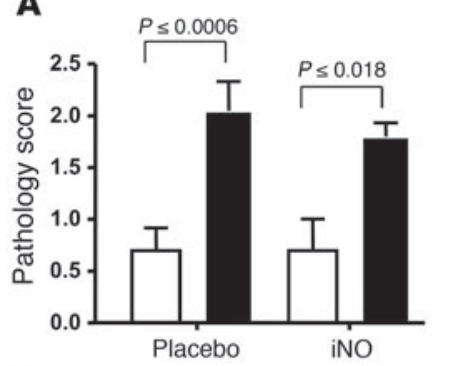

C

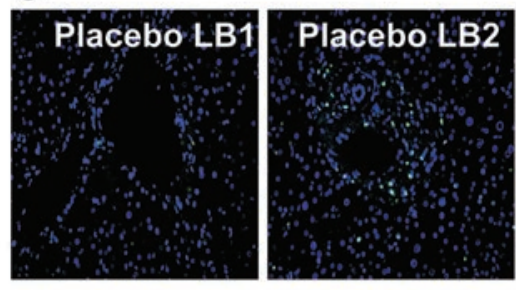

D

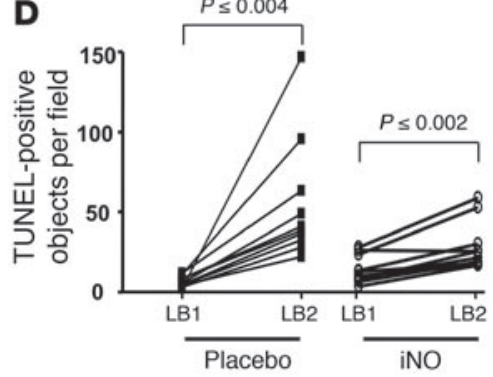

B

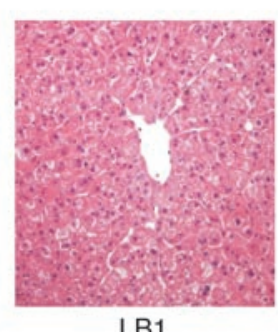

LB1

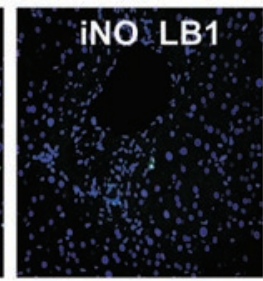

E

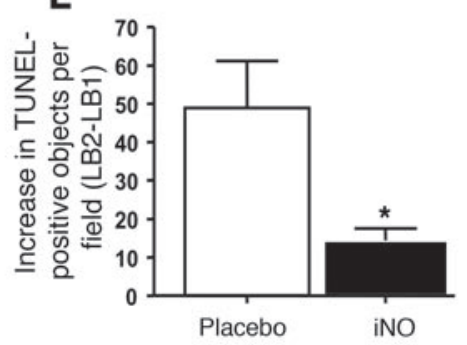

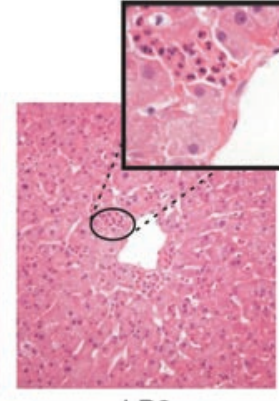

LB2

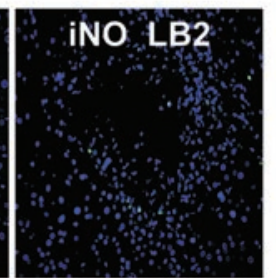

iNO
Figure 2

iNO decreases reperfusion-dependent hepatic cell death. (A) Histopathologic scoring of hepatic tissue samples before (white bars) and 1 hour after reperfusion (black bars). $P$ values represent significance calculated by paired $t$ test. (B) Representative $\mathrm{H} \& \mathrm{E}$-stained sections indicating increased injury in LB2. Original magnification, $\times 25$. The circled area is shown at a higher magnification $(\times 100)$ in the inset and shows increased PMN infiltration adjacent to the hepatic vein (zone 3). (C) Representative fluorescence micrographs showing changes in TUNELpositive cells (green); blue staining: DAPI. Original magnification, $\times 25$. (D) Paired changes in TUNELpositive objects in liver biopsies before (LB1) and 1 hour after reperfusion (LB2). $P$ values represent significance calculated by paired $t$ test. (E) Average reperfusion-dependent increases in TUNEL-positive objects. ${ }^{*} P \leq 0.0005$ relative to placebo.
iNO does not affect PMN infiltration but inhibits IR-induced hepatic cell death. Histopathologic evaluation of liver biopsy sections revealed a significant increase in injury 1 hour after reperfusion in both placebo and iNO groups (Figure 2, A and B), characterized by cytoplasmic vacuolization, nuclear pyknosis, sinusoidal dilatation, and PMN infiltration. This injury was localized primarily to the perivenular zones (Figure 2B). Similar trends were observed when inflammation was assessed specifically by scoring for PMN infiltration or myeloperoxidase (MPO) expression (Supplemental Figure 2 , A and B). Although reperfusion increased histopathologic injury scoring, PMN scoring, and MPO levels, the increases were not significantly different between placebo and iNO groups (Supplemental Figure 2, C-E). Despite the increase in PMNs, no significant differences in expression of the adhesion molecules P-selectin, E-selectin, VCAM-1, or ICAM-1 (Supplemental Figure 3, A-D) were observed upon reperfusion in either placebo or iNO groups. Concomitant with increased PMNs, reperfusion resulted in increased apoptosis (indicated by TUNEL staining) in both the placebo and iNO groups (Figure 2, C and D) that was centered around the central vein and consistent with hepatocyte apoptosis (Supplemental Figure 5). The magnitude of increased TUNEL staining was significantly attenuated $(-75 \%)$ in patients receiving iNO (Figure $2 \mathrm{E}$ ), suggesting a cytoprotective mechanism of action.

Effects of iNO on indices of oxidative and nitrosative stress. To further evaluate the underlying mechanisms of cytoprotection by iNO, protein carbonyl, 3-nitrotyrosine, liver and plasma xanthine oxidoreductase (XOR), and liver heme oxygenase-1 (HO1) were measured as either indices or modulators of oxidative/nitrosative LB1 and 3 LB2 specimens showed positive immunoreactivity for 3-nitrotyosine. Similar results were obtained using immunofluorescence approaches to measure 3-nitrotyrosine in formalin-fixed liver biopsies (data not shown). Note that a qualitative analysis of these data is presented here since the majority of samples measured were negative for 3-nitrotyrosine staining. An important source of ROS and effector of NO bioavailability is $\mathrm{XOR}$, as both xanthine oxidase and xanthine dehydrogenase are capable of reactive species production (22). Reperfusion resulted in similar increases in XOR activity in the plasma of placebo and iNO patients (Figure 3A), whereas no change in total hepatocellular XOR protein levels was observed (Figure 3B). Similarly, no change in hepatic expression of HO-1 was observed (Supplemental Figure 4B). Finally, hepatic expression of eNOS was measured, since NO production from this isoform has been shown to protect the liver against IR injury and eNOS expression reported to be downregulated in liver transplantation patients $(7,8)$. In our patient cohort, however, in the placebo group, 1-hour reperfusion had little effect on eNOS expression (Figure 3C). Surprisingly, in the iNO group eNOS expression significantly ( $80 \%)$ decreased after reperfusion (Figure 3, C and D). In contrast, iNO had no effect on expression of iNOS, which similar to previous studies did not change within 1 hour of reperfusion (Supplemental Figure 4C).

Effects of $i N O$ on circulating NO derivative levels. How iNO mediates extrapulmonary effects remains unclear. In order to gain insights into the possible biochemical intermediates and their potential contribution to iNO-mediated extrapulmonary mechanisms, we measured circulating levels of plasma and rbc nitrate, nitrite, $\mathrm{SNO}, \mathrm{XNO}$, and rbc HbNO (Figure 4). Analysis by 2-way repeated- 

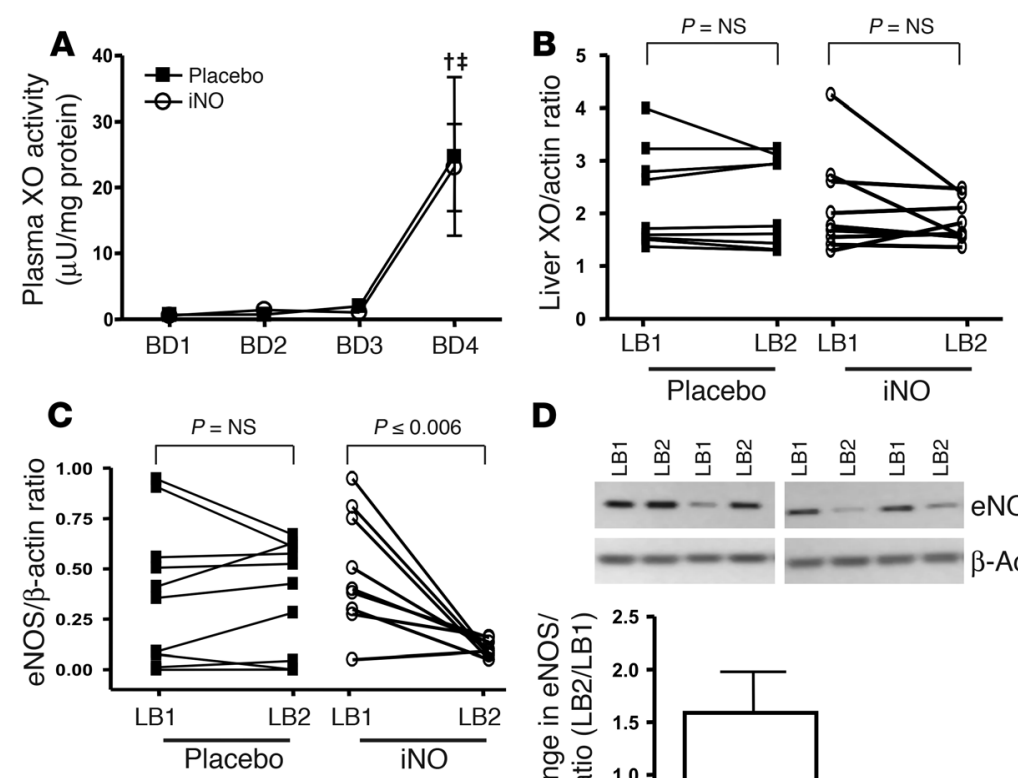

D
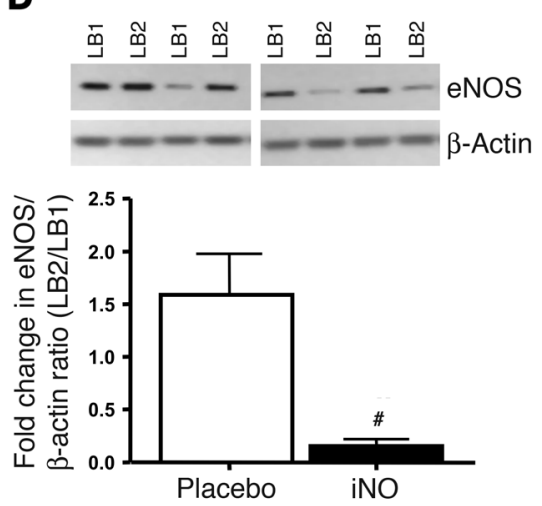

\section{Figure 3}

Effects of iNO on ROS and RNS metabolism. (A) Plasma XOR activities as a function of blood draws during surgery. ${ }^{\dagger} P \leq 0.05$, BD4 relative to BD1 for placebo; $¥ P=<0.001$, BD4 relative to $\mathrm{BD} 1$ for iNO by repeated-measures ANOVA with Tukey post-hoc analysis. (B) Changes in hepatocellular expression of XOR (normalized to $\beta$-actin) before and 1 hour after reperfusion. (C) Changes in hepatic expression of eNOS (normalized to $\beta$-actin) before and 1 hour after reperfusion. $P$ values represent significance calculated by paired $t$ test. $n=10$, placebo; $n=9$, iNO. Inset in $\mathbf{D}$ shows representative Western blots showing changes in eNOS in paired (LB1-LB2) samples from 2 patients in each group. (D) Average fold change in eNOS expression. ${ }^{\#} P \leq 0.004$ relative to placebo. measures ANOVA indicated that iNO selectively (relative to placebo) increased circulating levels of plasma nitrate, nitrite, and $\mathrm{rbc} \mathrm{HbNO}$. It should be noted that the absolute concentrations of SNO and XNO in plasma or rbc were between 10- and 20-fold less than corresponding nitrite levels, which in turn were greater than 2 orders of magnitude lower than corresponding nitrate levels. Post-test analysis revealed significant differences between placebo and iNO at blood draw 1 (BD1), $-2,-3$, and -4 for plasma nitrite and $\mathrm{rbc} \mathrm{HbNO}$ and at $\mathrm{BD} 4$ for plasma nitrate and rbc nitrate. No significant differences between placebo and iNO for plasma and rbc SNO nor XNO were observed.

Additionally, paired arterial and venous samples at each blood draw were collected to gain insight into which NO derivative (NOx) species may be consumed across the microcirculation. No differences in plasma/rbc A-V gradients of SNO or XNO were observed between placebo and iNO groups at any blood draw (data not shown). At BD 3 (immediately before reperfusion), significantly different (between placebo and iNO) A-V gradients were observed only in plasma nitrite (Figure 4J).

\section{Discussion}

Therapeutic attenuation of IR injury is anticipated to improve liver function after transplantation. In this study we targeted NO, since decreased production of this free radical has been indicated in human liver transplantation and its administration shown to attenuate many features of IR injury, including inflammation and cytotoxicity in animal-based studies $(7,8,16)$. Robichaud et al. first introduced the concept that iNO can mediate NO-dependent effects in the extrapulmonary vasculature (11). This observation has since been reproduced and led to the general concept that iNO can replenish NO bioactivity in systemic vascular beds, which are characterized by a deficit in NO function and bioavailability $(12-15)$. We reasoned that iNO, with its acceptable safety profile and minimal effect on total peripheral resistance, represents a via- ble therapy to limit hepatic IR injury in human liver transplantation and evaluated the hypothesis that iNO abrogates IR-induced hepatocellular inflammation and cytotoxicity.

Importantly, use of iNO at $80 \mathrm{ppm}$ for the duration of surgery was well tolerated, did not result in any undesirable changes in cardiopulmonary performance (data not shown), caused no direct toxicity, nor did it predispose the patient to any secondary toxicities associated with pulmonary complications or infection. In fact, iNO decreased the necessity for platelet transfusion during surgery, although the mechanism of this effect remains unclear at this time. Treatment with iNO significantly decreased the time in the hospital, by approximately $10 \%$ (1.24 days on average) when adjusted for sex and cold ischemic time. Given that many factors ultimately lend themselves to a patient's hospital discharge, especially in the case of organ transplantation, this was a surprising finding and consistent with the overall conclusion that iNO improves the rate of recovery of liver function, translating to a more expedient departure from the hospital. Hospital discharge times are notable parameters, since lower lengths of stay may reduce health cost burdens and decrease the probability of nosocomially acquired infections. We chose to use survival analysis for this data despite the fact that all 20 patients survived the transplant (a predicted 1-year mortality rate of $5 \%-15 \%$ ) due to prespecification in the protocol, and adjustments for sex, race (nonsignificant), and cold ischemic time in the time in hospital analysis were set by clinicians independent of the clinical trial data. These findings also raise the possibility that iNO may allow use of donor livers with longer cold ischemic times, thus serving to potentially expand the donor organ pool available to transplantation.

A significant clinical finding in this study was that iNO improved the rate of functional recovery of the transplanted liver. Serum AST and ALT and coagulation times measuring both the intrinsic and extrinsic pathways improved at a faster rate in patients receiving iNO. It should be noted that changes in serum creatinine were not 

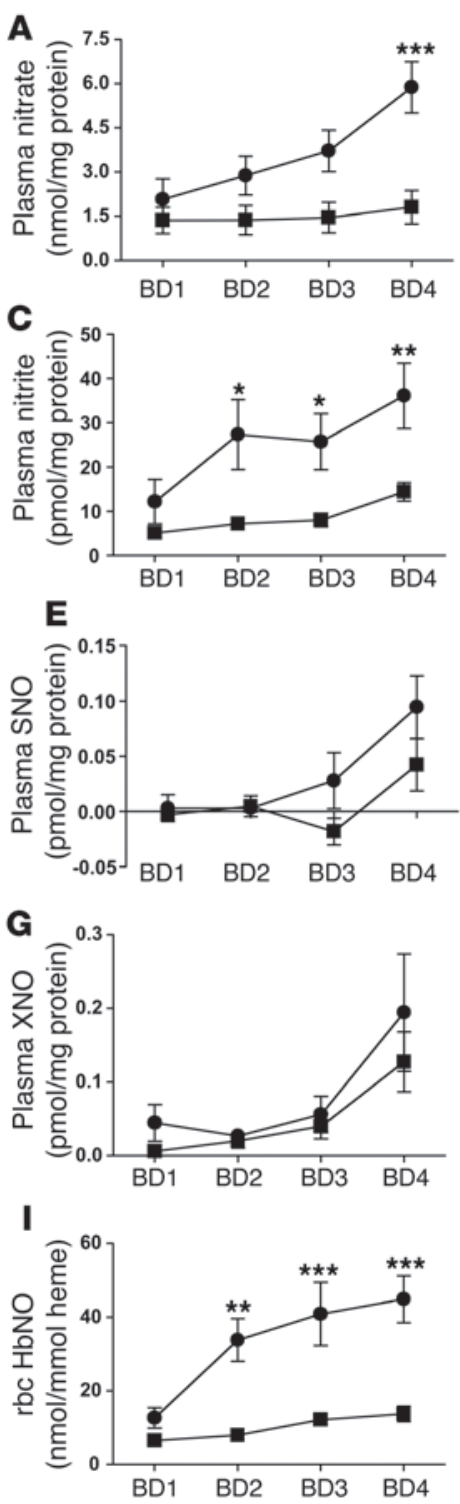
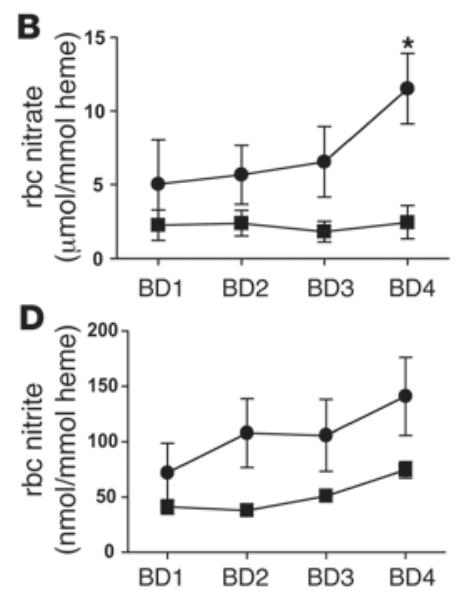

$\mathbf{F}$

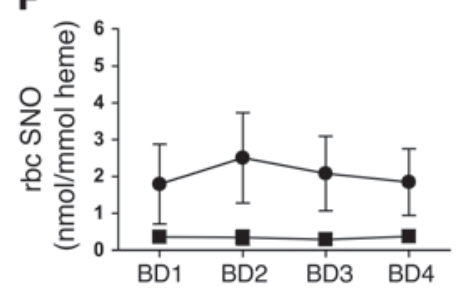

H
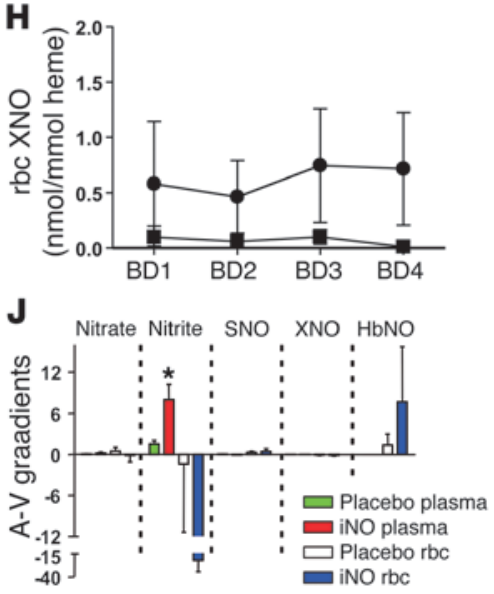

\section{Figure 4}

Changes in circulating NOx metabolites. Plasma (A, C, E, and $\mathbf{G})$ and $r b c(\mathbf{B}, \mathbf{D}, \mathbf{F}, \mathbf{H}$, and $\mathbf{I})$ of nitrate ( $\mathbf{A}$ and $\mathbf{B})$, nitrite (C and D), SNO (E and F), XNO (G and $\mathbf{H}$ ), and $\mathrm{HbNO}$ (I) are shown as a function of blood draw in placebo (filled squares) and iNO (filled circles) groups. Plasma NOx levels were normalized to protein. Absolute concentration ranges (minimum to maximal) were: nitrate $(\mu \mathrm{M})$, placebo $47.9 \pm 15.7$ to $54.7 \pm 19.2$, iNO $84.6 \pm 32.5$ to $140.3 \pm 24.5$; nitrite $(n M)$, placebo $202 \pm 38.4$ to $381.3 \pm 49.3$, iNO $462.2 \pm 181.13$ to $923.1 \pm 240$; SNO $(n M)$, placebo $-0.16 \pm 0.17$ to $1.3 \pm 0.66$, iNO $0.05 \pm 0.2$ to $2.3 \pm 0.66 ; \mathrm{XNO}(\mathrm{nM})$, placebo, $0.29 \pm 0.2$ to $3.5 \pm 0.58$, iNO $1.04 \pm 0.35$ to $4.7 \pm 1.9$. rbc NOx levels were normalized to heme. Absolute concentrations (in packed rbc) were: nitrate $(\mu \mathrm{M})$, placebo $31.7 \pm 12.5$ to $41.5 \pm 19.1$, iNO $80.3 \pm 48.3$ to $196.2 \pm 44.3$; nitrite $(n M)$, placebo $646.1 \pm 80.8$ to $1,219.5 \pm 131.7$, iNO $1,241.4 \pm 447.7$ to $2,533.3 \pm 681.1$; SNO $(\mathrm{nM})$, placebo $4.9 \pm 2.5$ to $6.2 \pm 2.2$, iNO $29.1 \pm 16.3$ to $40.7 \pm 18.2 ; \mathrm{XNO}(\mathrm{nM})$, placebo $0.19 \pm 0.15$ to $1.8 \pm 1.2$, iNO $7.3 \pm 4.9$ to $13.4 \pm 9.3$; $\mathrm{HbNO}$ $(\mathrm{nM})$, placebo $93.4 \pm 4.4$ to $242.8 \pm 32.9$, iNO- $222.9 \pm 53.2$ to $764.6 \pm 108.1$. Differences were assessed by 2-way repeated measures ANOVA and Bonferroni post-test. ${ }^{\star} P \leq 0.05,{ }^{\star \star} P \leq 0.01,{ }^{* \star \star} P \leq 0.001$ for placebo versus iNO at corresponding blood draw. (J) Plasma and rbc A-V gradients at BD3 for indicated NO intermediates. Concentration units for each NOx are as indicated in respective panels showing changes in individual NOx as a function of BD (A-I). ${ }^{\star} P \leq 0.001$ for placebo versus iNO for plasma nitrite. All values are mean \pm SEM; $n=10$, and $n=6-7$ for $\mathrm{HbNO}$. different (data not shown) between iNO and placebo patients, suggesting that differences in renal clearance are unlikely to account for the changes in measured liver function via transaminases. There are several factors that likely contribute to the enhanced rate of recovery of liver function. Discussed below is the potential of limiting cytotoxicity and inflammation, 2 responses integral in IR injury.

Inflammatory leukocyte-dependent tissue injury is a central feature of many IR injury models and in the liver is thought to mediate the second or late phase of injury (6). However, the precise role for leukocytes remains under investigation, with no role suggested in murine models of reduced-size liver transplantation in mice (1). Nevertheless, iNO has been shown to limit leukocyte accumulation in mesenteric IR models (11) and in skeletal muscle IR injury in humans undergoing knee surgery (15). These studies led to our primary hypothesis that iNO would attenuate leukocyte infiltration in the liver after reperfusion. Significant increases in PMN and hepatocellular injury were observed in the perivenular area (zone 3) within 1 hour of reperfusion, which was unaffected by iNO. A potential limitation in our study design was assessment of liver inflammation 1 hour after reperfusion, a time point that, as revealed in some models of IR injury, may not be long enough to allow observation of significant increases in adhesion molecules and subsequent PMN accumulation (15). However, an increase in PMN infiltration was observed and occurred independently of any changes in adhesion molecules, suggesting either that the donor liver expresses a sufficient complement of adhesion molecules (stimulated by harvesting, cold ischemia, etc.) or an adhesion molecule-independent (e.g., mechanical obtrusion) mechanism of leukocyte accumulation in the hepatic sinusoids (6). In either case, there was lack of efficacy with iNO in attenuating the initial events leading to inflammatory cell accumulation in the liver after organ reperfusion/transplantation.

iNO improved serum transaminase profiles, which generally indicate a reduction in hepatocellular injury. No differences in ALT or AST measurements immediately after surgery were observed, suggesting that changes in cell death per se were not directly linked to the initial release of serum transaminases. However, iNO did decrease apoptosis, raising the question of the link between 
cytotoxicity and the functional improvement in liver function. Typically, apoptosis is considered to be a physiological process that coupled with phagocytic clearance limits both necrotic cell death and the ensuing inflammatory response. However, apoptotic cells can evolve into necrotic cells and in some experimental liver injury models can directly trigger PMN infiltration as well (23). Study design limitations precluded assessment of necrosis and inflammation at later times, and it remains to be elucidated whether the decrease in apoptosis by iNO at 1 hour after reperfusion also downregulates the characteristic IR-induced cascade involving proinflammatory cytokine release and further PMN infiltration and cell death.

Low NO concentrations protect hepatocytes against apoptosis (9) via multiple potential mechanisms. A role for $\mathrm{HO}-1$ was excluded based on the fact that there were no changes in HO- 1 protein expression within 1 hour of reperfusion. In various liver IR models, ROS and RNS formation are thought to be central to cytotoxicity $(1,6)$. As expected, reperfusion was associated with a significant increases in plasma XOR activity. However, iNO had no effect on this or hepatic XOR expression, neither was oxidative protein damage (assessed by carbonyl and 3-nitrotyrosine formation) observed 1 hour after reperfusion, suggesting that iNO is not inducing oxidative stress over this time frame. These data suggest a trivial role for ROS scavenging, but it is important to note that various ROSs and RNSs can exist, which also differ in reactivity and biological targets, the latter of which may also change temporally (lowmolecular-weight thiol oxidation may precede protein oxidation, for example); thus, we cannot exclude a role for NO interactions with ROS and RNS in the hepatoprotective effects observed.

An intriguing observation was the effect of iNO on hepatic expression of eNOS. In the placebo group, eNOS increased minimally 1 hour after reperfusion, in contrast to a recent study with human liver transplantation patients that demonstrated a significant decrease (7). Unexpectedly, eNOS expression significantly decreased $(>80 \%)$ in patients receiving iNO. At first glance, these data appear to be in contrast to experimental studies definitively demonstrating that hepatic eNOS deficiency exacerbates IR injury (24). However, increased injury was not observed, and in fact, patients receiving iNO whose livers had lower eNOS expression demonstrated improved liver function. A key distinction between experimental studies and our study is that decreased eNOS was observed 1 hour after reperfusion, while prereperfusion levels were not different. The fact that eNOS loss was observed within 1 hour after reperfusion may indicate a rapid turnover/degradation type mechanism rather than blocking of eNOS protein synthesis. The stimulus for the rapid decrease in eNOS expression is not known. We hypothesize that the exposure of the liver to exogenous NO (via iNO-derived nitrite or other NO-containing species; see below) results in a downregulation of endogenous NO production via eNOS, consistent with a dynamic regulatory system. Indeed, precedent for such a process is provided by studies demonstrating that eNOS deficiency hypersensitizes cells to exogenous NO donors (25, 26). These concepts in the setting of IR injury, as well as any potential consequence on liver function, remain to be investigated.

We also collected blood samples throughout the operative period to gain insights into the possible NO-containing mediator that allows iNO to stimulate NO-dependent effects in extrapulmonary tissues. The distribution of different NO-metabolites showed nitrate to be the most concentrated, followed by nitrite and $\mathrm{HbNO}$, which in turn were present at greater levels than SNO and XNO. As expected, nitrate was formed to the greatest extent $(\mu \mathrm{M})$ and is generally considered as the ultimate inert end product of NO metabolism. Nitrate increased continuously with iNO exposure and likely reflects NO (and nitrite) oxidation to nitrate by oxyhemoglobin (27). Although nitrate per se is inert biologically, it can be reduced to nitrite by commensurable bacterial nitrate reductases in the oral cavity and in this way can modulate gastrointestinal and circulating NO bioactivity (28). In this study, however, nitrate formation was occurring directly in the circulation, precluding this potential pathway. Alternatively, nitrate reduction to nitrite and $\mathrm{NO}$ by XOR has been reported, although this reaction occurred with relatively slow kinetics and required high nitrate concentrations with reported $K_{\mathrm{m}}$ in the 5- to $10-\mathrm{mM}$ range (29). While we cannot exclude this pathway as a potential mechanism for nitrite and NO formation, maximal nitrate concentrations reached only $196.2 \pm 44.3 \mu \mathrm{M}$ with iNO exposure, suggesting that XOR-dependent reduction to nitrite is unlikely to play a significant role in nitrite and $\mathrm{NO}$ generation.

Although $\mathrm{HbNO}$ was also significantly elevated in the iNO group, it too is unlikely to be a direct mediator of NO delivery. The high affinity of deoxyhemoglobin for NO and high rate of oxyhemoglobin-dependent NO oxidation make it unlikely that $\mathrm{HbNO}$ directly transduces NO bioactivity out of the rbc and to the liver (30). Both plasma and rbc SNO can stimulate NO-dependent bioactivity $(31,32)$, and the antiinflammatory effects of iNO in a feline model of mesenteric IR injury were proposed to occur via increases in plasma $S$-nitrosoalbumin (16). However, in this study, continuous exposure ( $\sim 4$ hours) to iNO did not significantly change plasma or red cell SNO or XNO concentrations, consistent with previous studies that employed lower iNO exposure times (14), suggesting that plasma or $\mathrm{rbc}$ SNO/XNO are not the key mediators of iNO-dependent effects observed in this study. In addition, these results indicate that iNO in general at $80 \mathrm{ppm}$, for approximately 4 hours, either directly or via secondary NO metabolites (e.g., nitrite) (20), does not induce an overwhelming nitrosative stress, the latter typically being associated with significant formation of circulating SNO (31).

Of all the possible candidates, we hypothesize nitrite as being the most likely candidate for transducing the iNO stimulus to the liver. First, circulating nitrite concentrations were significantly increased in patients receiving iNO and together with significant $\mathrm{A}-\mathrm{V}$ gradients in plasma nitrite, but not other NO-containing metabolites, suggest that metabolism of this anion across the microcirculation is a dynamic process that as discussed below may result in NO generation. Nitrite formation was also significant in murine models in which iNO protected against myocardial IR injury (12). Nitrite consumption was observed across the forearm microvasculature in humans administered iNO and was associated with increased blood flow (14). More importantly, reaction mechanisms are being defined that demonstrate a hypoxia-sensitive reactivity of nitrite that forms $\mathrm{NO}$ and other NO-containing molecules $(18,19)$. This dynamic interplay between nitrite and $\mathrm{NO}$ under biological conditions is thought to underlie functional observations that reveal low-dose $(\mathrm{nM})$ reagent nitrite is protective in diseases characterized by ischemia and NO deficiency, including but not limited to hepatic and myocardial IR injury, stroke, and pulmonary hypertension $(17,18,20,33-35)$. The general model proposed includes an ischemia-sensitive mechanism that couples oxygen sensing to reduction of nitrite to NO. Candidates that link oxygen sensing to nitrite reduction include a deoxy ferrous heme 
protein (e.g., deoxyhemoglobin or hepatic heme proteins) (18). Alternative mechanisms proposed include acid disproportionation (acidic conditions arising from ischemia) and reduction of nitrite to NO by XOR, an enzyme proteolytically activated during ischemia (35-37), although XOR inhibition alone (independent of nitrite administration) generally protects against IR injury (22). Irrespective of the mechanism, NO formation appears to be criti$\mathrm{cal}$, since NO scavenging in experimental studies reverses the protective effects of nitrite $(17,35)$. In our study, circulating nitrite levels (in whole blood) increased in response to iNO and remained at a steady state of approximately $800 \mathrm{nM}$ (note that nitrate formation increased continuously, suggesting continuous nitrite oxidation to nitrate). This low submicromolar level of nitrite is within the range that conferred protection in animal models of IR injury, consistent with the proposal that iNO confers hepatic protection via nitrite. How iNO increases circulating nitrite concentrations is not known but likely involves recently described metalloproteindependent oxidation (e.g., ceruloplasmin) (38) and requires further investigation in the context of iNO-based therapies.

A critical element of the nitrite model is localized production of $\mathrm{NO}$ at or in the ischemic tissue, which can then stimulate a variety of effects (e.g., inhibiting apoptosis). Whereas this may be the case during reagent nitrite administration directly to the tissue, with iNO, the transplanted liver will only be exposed to nitrite upon reperfusion. In this scenario, nitrite may still produce $\mathrm{NO}$ during the initial stages of reperfusion and tissue hypoxia or do so via reactions with deoxygenating $\mathrm{rbc}(18)$. In this case, another protective mechanism of action may involve improved hepatic blood flow upon reperfusion. This would also suggest that the timing of iNO administration could be limited to the extent that the circulating nitrite levels reach a steady state. These and other possible dosing strategies remain to be tested.

The main limitation of the current study is the relatively small sample size. Although significance in time in hospital and liver function tests was observed, a larger trial is required to evaluate the potential of iNO to prevent IR injury in the transplanted liver. Also, and as discussed above, limiting the second liver biopsy to 1 hour after reperfusion may not have allowed assessment of all potential effects of iNO on inflammation (specifically cytokine release) and necrotic cell death. In addition, the choice of protein oxidation and XOR as indices of ROS/RNS metabolism may not have been appropriate indices for accurately assessing oxidative/ nitrosative stress. For example, lipid or thiol oxidation may be more prevalent than protein oxidation, and ROS sources other than XOR [e.g., NAD(P)H oxidase] may play a role in IR injury. Finally, a role for nitrated lipids, a novel class of NO-containing intermediates that can elicit NO signaling (39), as potential candidates for mediated extrapulmonary effects of iNO was not assessed in the current study. In summary, this "pilot" study clearly validates that preemptive administration of iNO to liver transplant recipients is safe (without toxicity) and establishes the potential for an efficacious approach that may limit IR injury and improve human liver function after transplantation.

\section{Methods}

Detailed methods are presented as online supplemental information.

Patient enrollment and study design. Twenty-seven adult patients ( $>18$ years of age) admitted consecutively to the University Hospital, University of Alabama at Birmingham (UAB), for liver transplantation were screened for participation in the study between December 2005 and May 2006. Of these,
20 patients were enrolled. Patients were excluded if consent was denied $(n=3)$, if undergoing dual transplantation (liver and kidney; $n=1)$, if under 19 years of age $(n=1)$, or if underlying pulmonary complications associated with hepatopulmonary syndrome were indicated in the recipient $(n=1)$. In addition, 1 patient was enrolled but did not receive a donor liver at UAB during the study. Further details describing clinical parameters used in exclusion criteria are presented in the Supplemental Data. Transplantation was performed without veno-veno bypass. Patients were randomly assigned to receive either placebo (nitrogen) or iNO (80 ppm). Unlabeled tanks containing NO or nitrogen gas were randomly numbered from 1 to 20 using an Internet-based random number generator (Pro 1.47; Segobit Software). Subsequently, sealed envelopes containing these numbers were prepared and randomly selected (upon receipt of patient informed consent), again using an Internet-based random number generator. Study investigators were blinded to the assignment of placebo or iNO, and all protocols were approved by the institutional review board of the University of Alabama at Birmingham. Figure 1A displays the study protocol. Upon achieving a stable hemodynamic status after the induction of anesthesia, BD1 was taken and followed by the initiation of either the placebo or iNO gas, with it being continued until 1 hour after reperfusion. Three subsequent blood draws were taken: $\mathrm{BD} 2$ taken approximately 1 hour after BD1; BD3 immediately before portal vein reperfusion; and BD4 1 hour after dual hepatic artery and portal vein reperfusion. Wedge biopsies from the right lobe of the donor liver were collected before reperfusion (LB1) and 1 hour after dual reperfusion (LB2). Clinical assessment of liver function after transplantation were evaluated by measuring serum levels of ALT and AST and coagulation times, specifically PT and PTT.

Blood collection and processing. With each blood draw, paired arterial (brachial artery) and venous (jugular vein) samples were collected. Due to the lability of different NOx, samples were processed at the bedside to stabilize plasma and rbc NOx. The different NOx measured included nitrate, nitrite, $\mathrm{SNO}, \mathrm{XNO}$, and $\mathrm{HbNO}$ by $\mathrm{NO}$ chemiluminescence as previously described $(31,40,41)$. All methods were validated independently in our laboratory with limits of detection in rbc or plasma being $100 \mathrm{nM}$ for nitrate and $25 \mathrm{nM}$ for other NOx (see Supplemental Data for detailed protocols). Venous plasma was also collected for measurement of cGMP (ELISA; Cayman Chemical) and XOR activities (by HPLC). Detailed methods are provided in Supplemental Data.

Liver biopsy processing. Upon retrieval, liver biopsies were immediately divided into equal sections and either fixed in formalin or snap-frozen in liquid nitrogen. Formalin-fixed samples were sectioned $(5 \mu \mathrm{m})$ and used for histochemical evaluation of hepatocellular injury, immunofluorescence detection of MPO, and TUNEL staining (Promega). Frozen samples were used for Western blotting, dot blotting, and ELISA-based assays. Liver expression of XOR, HO-1, eNOS, iNOS, P-selectin, and E-selectin was determined by Western blot analysis and normalized to $\beta$-actin expression. Liver expression of VCAM- 1 and ICAM- 1 was determined by ELISA. 3-Nitrotyrosine content was measured by dot blot on frozen samples and by immunofluorescence on formalin-fixed samples. Hepatocellular apoptosis was determined using the TUNEL assay.

Histopathology scoring. Morphologic evaluation of 5- $\mu \mathrm{m}$ formalin-fixed liver sections stained with $\mathrm{H} \& \mathrm{E}$ was performed by 2 independent pathologists blinded to the study protocol and according to the following scoring criteria: 0 , no hepatocellular damage; 1 , mild injury characterized by cytoplasmic vacuolization and focal nuclear pyknosis; 2 , moderate injury with dilated sinusoids, cytosolic vacuolization, and blurring of intercellular borders; 3 , moderate to severe injury with coagulative necrosis, abundant sinusoidal dilation, rbc extravasation into hepatic chords, and hypereosinophilia and margination of neutrophils; 4 , severe necrosis with loss of hepatic architecture, disintegration of hepatic cords, hemorrhage, and 
neutrophil infiltration. Criteria to specifically evaluate PMN infiltration (0, zero; 1 , minimal; 2 , mild; 3 , moderate; 4 , severe) were also used.

Statistics. Differences between measured parameters in patients receiving either placebo or iNO were first tested for normality (D'Agostino and Pearson omnibus normality test) and then evaluated by either the Mann-Whitney $U$ test (denoted by *) if the data was nonnormal or by the unpaired equal variance $t$ test (denoted by \#) if normal. For NOx, repeated-measures ANOVA with Bonferroni post-tests were used to assess time-dependent changes and differences between placebo and iNO groups at each time point. Time in hospital was analyzed using Cox proportional hazards regression analysis; subjects were analyzed per protocol adjusted for sex and cold ischemic time based upon a priori clinical advice. Unless indicated, all analyses represent $n=10$ for placebo and $n=10$ for iNO groups. In some instances due to limited sample, the number of replicates was less than 10 , as indicated in figure legends. All values represent mean \pm SEM. Analysis was performed using GraphPad Prism software.

1. Urakami, H., and Grisham, M.B. 2006. Divergent roles of superoxide and nitric oxide in reduced-size liver ischemia and reperfusion injury: implications for partial liver transplantation. Pathophysiology. 13:183-193.

2. Jaeschke, H., Smith, C.V., and Mitchell, J.R. 1988. Reactive oxygen species during ischemia-reflow injury in isolated perfused rat liver. J. Clin. Invest. 81:1240-1246.

3. Fondevila, C., Busuttil, R.W., and Kupiec-Weglinski,J.W. 2003. Hepatic ischemia/reperfusion injury - a fresh look. Exp. Mol. Pathol. 74:86-93.

4. Fellstrom, B., et al. 1998. Postischemic reperfusion injury and allograft arteriosclerosis. Transplant Proc. 30:4278-4280.

5. Kupiec-Weglinski, J.W., and Busuttil, R.W. 2005. Ischemia and reperfusion injury in liver transplantation. Transplant Proc. 37:1653-1656.

6. Jaeschke, H., and Hasegawa, T. 2006. Role of neutrophils in acute inflammatory liver injury. Liver Int. 26:912-919.

7. Varadarajan, R., et al. 2004. Nitric oxide in early ischaemia reperfusion injury during human orthotopic liver transplantation. Transplantation. 78:250-256.

8. Duranski, M.R., et al. 2006. Genetic overexpression of eNOS attenuates hepatic ischemia-reperfusion injury. Am. J. Physiol. Heart Circ. Physiol. 291:H2980-H2986.

9. Li, J., and Billiar, T.R. 1999. Nitric oxide. IV. Determinants of nitric oxide protection and toxicity in liver. Am. J. Physiol. 276:G1069-G1073.

10. Griffiths, M.J., and Evans, T.W. 2005. Inhaled nitric oxide therapy in adults. N. Engl. J. Med. 353:2683-2695.

11. Fox-Robichaud, A., et al. 1998. Inhaled NO as a viable antiadhesive therapy for ischemia/reperfusion injury of distal microvascular beds. J. Clin. Invest. 101:2497-2505.

12. Hataishi, R., et al. 2006. Inhaled nitric oxide decreases infarction size and improves left ventricular function in a murine model of myocardial ischemia-reperfusion injury. Am. J. Physiol. Heart Circ. Physiol. 291:H379-H384.

13. Gianetti, J., et al. 2004. Supplemental nitric oxide and its effect on myocardial injury and function in patients undergoing cardiac surgery with extracorporeal circulation. J. Thorac. Cardiovasc. Surg. 127:44-50

14. Cannon, R.O., 3rd, et al. 2001. Effects of inhaled nitric oxide on regional blood flow are consistent

\section{Acknowledgments}

Financial support for this study was provided by INO Therapeutics.

Received for publication February 19, 2007, and accepted in revised form June 12, 2007.

Address correspondence to: John D. Lang, Department of Anesthesiology, University of Washington, VA Puget Sound Health Care System, 1660 South Columbian Way, Seattle, Washington 98108-1597, USA. Phone: (206) 764-2854; Fax: (206) 764-2914; E-mail: jdlang@ u.washington.edu. Or to: Rakesh P. Patel, Department of Pathology, University of Alabama at Birmingham, 901 19th Street South, BMR-2, Room 302, Birmingham, Alabama 35294, USA. Phone: (205) 9759225; Fax: (205) 934-7447; E-mail: rakeshp@uab.edu.

John D. Lang Jr. and Rakesh P. Patel are co-senior authors. with intravascular nitric oxide delivery. J. Clin. Invest. 108:279-287. doi:10.1172/JCI200112761.

15. Mathru, M., Huda, R., Solanki, D.R., Hays, S., and Lang, J.D. 2007. Inhaled nitric oxide attenuates reperfusion inflammatory responses in humans. Anesthesiology. 106:275-282.

16. Ng, E.S., et al. 2004. Enhanced S-nitroso-albumin formation from inhaled NO during ischemia/ reperfusion. Circ. Res. 94:559-565.

17. Duranski, M.R., et al. 2005. Cytoprotective effects of nitrite during in vivo ischemia-reperfusion of the heart and liver. J. Clin. Invest. 115:1232-1240. doi:10.1172/JCI200522493.

18. Gladwin, M.T., et al. 2006. Nitrite as a vascular endocrine nitric oxide reservoir that contributes to hypoxic signaling, cytoprotection, and vasodilation. Am. J. Physiol. Heart Circ. Physiol. 291:H2026-H2035.

19. Gladwin, M.T., et al. 2005. The emerging biology of the nitrite anion. Nat. Chem. Biol. 1:308-314.

20. Bryan, N.S., et al. 2004. Cellular targets and mechanisms of nitros(yl)ation: an insight into their nature and kinetics in vivo. Proc. Natl. Acad. Sci. U. S. A. 101:4308-4313.

21. McMahon, T.J., and Doctor, A. 2006. Extrapulmonary effects of inhaled nitric oxide: role of reversible S-nitrosylation of erythrocytic hemoglobin. Proc. Am. Thorac. Soc. 3:153-160.

22. Zweier, J.L., and Talukder, M.A. 2006. The role of oxidants and free radicals in reperfusion injury. Cardiovasc. Res. 70:181-190.

23. Jaeschke, H., et al. 1998. Activation of caspase 3 (CPP32)-like proteases is essential for TNF-alphainduced hepatic parenchymal cell apoptosis and neutrophil-mediated necrosis in a murine endotoxin shock model. J. Immunol. 160:3480-3486.

24. Kawachi, S., et al. 2000. Nitric oxide synthase and postischemic liver injury. Biochem. Biophys. Res. Commun. 276:851-854.

25. Kevil, C.G., et al. 2004. Intercellular adhesion molecule-1 (ICAM-1) regulates endothelial cell motility through a nitric oxide-dependent pathway. J. Biol. Chem. 279:19230-19238.

26. Hussain, M.B., Hobbs, A.J., and MacAllister, R.J. 1999. Autoregulation of nitric oxide-soluble guanylate cyclase-cyclic GMP signalling in mouse thoracic aorta. Br. J. Pharmacol. 128:1082-1088.

27. Gladwin, M.T., Crawford, J.H., and Patel, R.P. 2004. The biochemistry of nitric oxide, nitrite, and hemoglobin: role in blood flow regulation. Free Radic. Biol. Med. 36:707-717.
28. Lundberg, J.O., Weitzberg, E., Cole, J.A., and Benjamin, N. 2004. Nitrate, bacteria and human health. Nat. Rev. Microbiol. 2:593-602.

29. Li, H., Samouilov, A., Liu, X., and Zweier, J.L. 2003. Characterization of the magnitude and kinetics of xanthine oxidase-catalyzed nitrate reduction: evaluation of its role in nitrite and nitric oxide generation in anoxic tissues. Biochemistry. 42:1150-1159.

30. Azizi, F., et al. 2005. Rates of nitric oxide dissociation from hemoglobin. Free Radic. Biol. Med. 39:145-151.

31. Crawford, J.H., et al. 2004. Transduction of NObioactivity by the red blood cell in sepsis: novel mechanisms of vasodilation during acute inflammatory disease. Blood. 104:1375-1382.

32. McMahon, T.J., et al. 2002. Nitric oxide in the human respiratory cycle. Nat. Med. 8:711-717.

33. Hunter, C.J., et al. 2004. Inhaled nebulized nitrite is a hypoxia-sensitive NO-dependent selective pulmonary vasodilator. Nat. Med. 10:1122-1127.

34. Pluta, R.M., Dejam, A., Grimes, G., Gladwin, M.T., and Oldfield, E.H. 2005. Nitrite infusions to prevent delayed cerebral vasospasm in a primate model of subarachnoid hemorrhage. JAMA. 293:1477-1484.

35. Webb, A., et al. 2004. Reduction of nitrite to nitric oxide during ischemia protects against myocardial ischemia-reperfusion damage. Proc. Natl. Acad. Sci. U. S. A. 101:13683-13688.

36. Li, H., Samouilov, A., Liu, X., and Zweier, J.L. 2001. Characterization of the magnitude and kinetics of xanthine oxidase-catalyzed nitrite reduction. Evaluation of its role in nitric oxide generation in anoxic tissues. J. Biol. Chem. 276:24482-24489.

37. Zweier,J.L., Wang, P., Samouilov, A., and Kuppusamy, P. 1995. Enzyme-independent formation of nitric oxide in biological tissues. Nat. Med. 1:804-809.

38. Shiva, S., et al. 2006. Ceruloplasmin is a NO oxidase and nitrite synthase that determines endocrine $\mathrm{NO}$ homeostasis. Nat. Chem. Biol. 2:486-493.

39. Schopfer, F.J., et al. 2005. Fatty acid transduction of nitric oxide signaling. Nitrolinoleic acid is a hydrophobically stabilized nitric oxide donor. J. Biol. Chem. 280:19289-19297.

40. Bryan, N.S., et al. 2005. Nitrite is a signaling molecule and regulator of gene expression in mammalian tissues. Nat. Chem. Biol. 1:290-297.

41. Wang, X., et al. 2006. Measurement of nitric oxide levels in the red cell: validation of tri-iodide-based chemiluminescence with acid-sulfanilamide pretreatment. J. Biol. Chem. 281:26994-27002. 